\title{
Interpreting Pedestrian Behaviour by Visualising and Clustering Movement Data
}

\author{
Gavin McArdle ${ }^{1}$, Urška Demšar ${ }^{2}$, Stefan van der Spek $^{3}$, and Seán McLoone ${ }^{4}$ \\ 1 National Centre for Geocomputation, National University of Ireland Maynooth, \\ Maynooth, Co. Kildare, Ireland \\ gavin.mcardle@nuim.ie \\ 2 Centre for GeoInformatics, School for Geography and Geosciences, \\ University of St Andrews, Fife, Scotland, UK \\ urska.demsar@st-andrews.ac.uk \\ 3 Department of Urbanism, Faculty of Architecture, \\ Delft University of Technology, Delft, the Netherlands \\ s.c.vanderSpek@tudelft.nl \\ 4 Department of Electronic Engineering, National University of Ireland Maynooth, \\ Maynooth, Co. Kildare, Ireland \\ sean.mcloone@eeng.nuim.ie
}

\begin{abstract}
Recent technological advances have increased the quantity of movement data being recorded. While valuable knowledge can be gained by analysing such data, its sheer volume creates challenges. Geovisual analytics, which helps the human cognition process by using tools to reason about data, offers powerful techniques to resolve these challenges. This paper introduces such a geovisual analytics environment for exploring movement trajectories, which provides visualisation interfaces, based on the classic space-time cube. Additionally, a new approach, using the mathematical description of motion within a space-time cube, is used to determine the similarity of trajectories and forms the basis for clustering them. These techniques were used to analyse pedestrian movement. The results reveal interesting and useful spatiotemporal patterns and clusters of pedestrians exhibiting similar behaviour.
\end{abstract}

Keywords Geovisual Analysis, Clustering, Space-time Cube, Movement Data Analysis

\section{Introduction}

Movement of individual objects such as animals, humans, human-operated objects or complex natural phenomena represent one of the main processes in the physical environment. For the purposes of data representation and modeling, such moving objects are considered as entities, whose position and/or geometric attributes change over time [1]. Despite the diversity of sources of movement data, fundamentally the same process is captured: how objects move through the basic framework of the physical world, defined by geographic space and time.

While the ubiquitous nature of location technologies has created issues for analysing movement data, specifically for pattern recognition and path predic- 
tion [2], it also provides an opportunity to understand how moving objects interact with each other and their environment. Due to interest in understanding human spatial behaviour, pedestrian mobility has long been an active research area in location based services [3], emergency management [4], determining infrastructural requirements [5] and urban planning [6]. Within urban planning and design, the vitality of a city is not generally measured using spatial analysis but by quantifying the number of people visiting the city and the economic value that brings. However, understanding the spatial and temporal aspects of the city, for example, the time spent and routes used by pedestrians can enhance a city's vitality. Current techniques in urban analysis, map the city based on its morphology, but questions about how the city network is used still remain. By analysing the activity patterns of individual pedestrians, repetitive behaviour and similar goals can be identified. This information can be translated into designs which address the requirements of the population, which is a key goal in urban design [6], [7].

This paper introduces a geovisual analytics tool for trajectory exploration, which contains novel techniques to help analysts comprehend movement datasets by visually representing the temporal and spatial aspects of movement simultaneously. Principle routes and frequent stopping locations can be determined. A new approach for identifying clusters of similar behaviour, also forms part of this research. A case study, using pedestrian data collected in the city of Delft in the Netherlands demonstrates these techniques.

This paper is structured as follows; the next section provides some related work in the area of movement data visualisation and analysis. Section 3 presents the novel visualisation and analysis techniques used to cluster movement data. A case study of pedestrian data, which reveals interesting spatiotemporal patterns and classifies pedestrians based on behaviour, is presented in Section 4. The paper concludes with a discussion and future work in Sections 5 and 6 .

\section{Visualisation and Analysis of Trajectory Data}

Driven by the increase in the quantity of movement data, this section examines questions regarding techniques to represent, interpret, analyse and compare movement data.

\subsection{Trajectory Representation and Visualisation}

The path of a moving object is usually represented as a trajectory which is a sequence of positions in a two-dimensional (2D) geographic environment with time stamps [8], i.e. $T=\left(x_{1}, y_{1}, t_{1}\right), \ldots,\left(x_{n}, y_{n}, t_{n}\right)$ for some $n$, such that $\left(x_{i}, y_{i}\right)$ is the measured geographic location of the moving object at time $t_{i}$. Often trajectories have associated attribute data, which can be static, if the attribute has the same value for the object regardless of its position, (e.g. object type) or dynamic, if the attribute changes over time (e.g. attributes which describe 
physical properties of movement, such as velocity and acceleration). Other interpretations conceptualise movement as a sequence of moves and stops [27], or activity chains [9]. A taxonomy for describing common movement patterns such as convergence, divergence and concurrence in space and time as well as behavioural patterns including pursuit, play and flocking activities which can be used to classify trajectories, has also been proposed [10].

For visual analysis, the complete trajectory can be represented on a 2D map, however, this removes the temporal aspect, as the sequence in which locations were visited is removed. While animation can be included, it is not possible to interpret the entire spatiotemporal extent of trajectories using this technique. A series of separate maps representing distinct time periods can show the progression of moving entities [11]. This so-called situation-oriented point-of-view offers a discrete rather than a continuous view of the movement and contrasts with a trajectory-oriented point of view which shows each trajectory throughout its life cycle. Trajectory-oriented visualisations are valuable when studying movement patterns, however in order to visually analyse the temporal patterns of trajectories, a technique capable of representing time is required.

The Space-time Cube (STC), which originates from Time Geography, is a visual technique for representing a trajectory-oriented point of view, incorporating the temporal elements of trajectories [12], [13]. In a web-based STC, the geographic coordinates appear on the $\mathrm{x}$-axis and $\mathrm{y}$-axis, while time is represented along the orthogonal z-axis. Time increases along this axis so that more recently occurring events appear above older events. Using this approach, a trajectory can be plotted as a three-dimensional (3D) polyline in the STC. While the STC is not a new concept, due to technological advances it has recently gained interest among the geovisual analytics research community for visual exploration of trajectories [14], [15]. A STC is suitable for simultaneously visualising a relatively low number of trajectories. When that number increases, the STC suffers from occlusion and cluttering, making it difficult to identify trends [16]. Usually aggregation, for example, using kernel densities [17] or generating clusters of the most salient patterns [18] can resolve this. We extend this idea with a new approach for clustering trajectories based on the projection of trajectories on the sides of the STC, described in Section 3.

\subsection{Trajectory Similarity}

One of the most important tasks for trajectory analysis is the identification of similar trajectories to reveal significant patterns. For example, it is used in animal ecology to estimate migration routes [19] and in meteorology to predict hurricane movement [20]. In urban planning, planners are interested in spatiotemporal patterns of pedestrians which requires using a large set of individual movement data over a long period.

Methods for identifying groups of similar trajectories are mostly based on their geometric similarity in 2D geographic space. Examples use the Euclidian distance [21], the Hausdorff Metric [22] and the Fréchet Distance Metric [23]. Dynamic Time Warping [24] considers the temporal aspect by stretching the 
time axis in order to identify similarities in trajectory shape. This allows a comparison of trajectories which span different periods and spatial regions. Spatial transformations can also realign trajectories for better comparison. The Longest Common Substring (LCSS) approach does not consider the entire trajectory but instead finds similarities between substrings [25]. Edit Distance on Real Sequence (EDR), which measures the number of operations (insert, delete or replace) required to transform one trajectory to another, extends this by assigning penalties to the gaps between two matched sub-trajectories [26]. Similarity is also measured by examining the places and locations where stops occur [27]. This approach has also been extended to consider places where entities move slowly as significant [28]. Additional attributes of movement (speed, acceleration, duration and direction) are also used to determine similarity [1].

The research presented in this article follows this trend. We augment a geovisual environment, based on the STC, with a new and effective technique for identifying salient clusters of trajectories. This novel approach relies on the physical decomposition of movement in orthogonal directions. Each trajectory is then described in terms of speed and acceleration at each point in each direction before similarity measures and clustering are applied. These techniques, as well as a description of the geovisual analysis environment are discussed in the next section.

\section{Trajectory Exploration Environment}

In the environment introduced here, two visualisation components are used to analyse movement data. The first element visualises trajectories in a STC where a virtual globe represents the spatial aspect and the second component plots trajectory paths decomposed into latitude and longitude to enable comparison. Additionally, powerful analysis tools cluster trajectories based on a novel similarity metric described in Section 3.3.

\subsection{Web-based Space-time Cube}

The principle visualisation component is a STC draped over a virtual globe (Google Earth), which provides a pseudo 3D view of the Earth. The third dimension represents the terrain of the underlying landscape. As demonstrated in figure 1, we manipulate the z-axis to represent time by draping a STC over the elevation surface (while the z-axis is a sum of elevation and trajectory time stamp, this can be changed). The trajectories therefore appear as lines floating above the surface of the Earth. The further from the surface a point on the trajectory appears, the more recent the observation. Time can be represented using different paradigms: each trajectory can start at a position relative to its time stamp in the overall dataset or each trajectory can begin their path at the Earth's surface.

Figure 1 also shows the visualisation interface. The bridge-like structures visible on the surface of the Earth represent trajectories. The points, connecting 


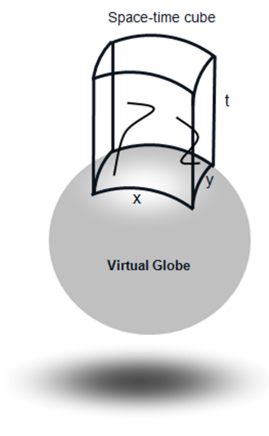

(a)

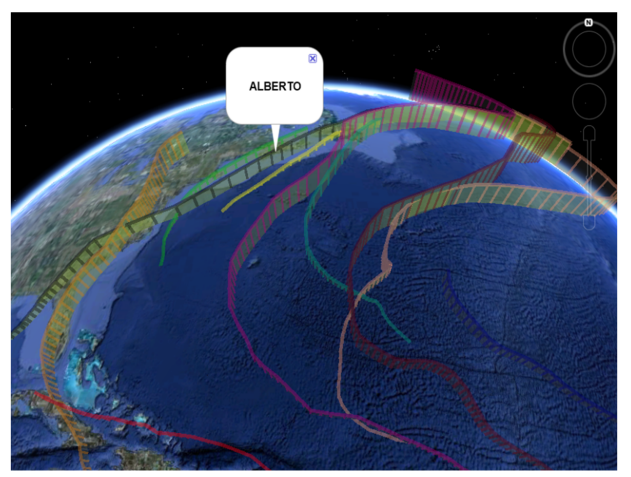

(b)

Fig. 1. (a) A STC containing trajectories draped over a virtual globe. (b) A STC containing hurricane trajectories shown in Google Earth

to the ground, along the trajectory are the points where the entity's location was recorded. Colours distinguish individual trajectories. The user can change the viewing angle, zoom level, interact with and identify spatial content. Using Google, the analyst can take advantage of Google Street View and Google Maps to obtain additional spatial information about the study area.

Through visual exploration, the analyst can obtain new information about the trajectories being studied. For example, it is possible to gauge the speed of entities (using the slope of the 3D polylines representing trajectories), the sequence in which locations are visited (by examining the position of the polyline of the z-axis relative to the Earth's surface) and compare the progress of entities using the same route (by examining the distance from the Earth's surface of the polylines representing the entities). Additionally, stopping positions can be determined by identifying areas with a large number of recordings in which the $\mathrm{z}$-axis position of the polyline changes, indicating time has progressed but the entity's spatial position has not altered.

\subsection{Physical decomposition of movement in space-time cube}

As described above, the path of a moving object in the STC is approximated as a series of straight-line segments between sampled points $\left(x_{i}, y_{i}, t_{i}\right)$ and $\left(x_{i+1}, y_{i+1}\right.$, $t_{i+1}$ ) [8]. Each trajectory is therefore represented by a continuous 3D polyline. This is combined with a principle stemming from classical mechanics: the physical description of motion in an inertial frame of reference. In our context, we are interested in the mathematical description of this motion, which is normally done via vector analysis in the inertial frame of reference [29]. In classical mechanics, the inertial frame of reference is represented by a 3D Cartesian coordinate system located at an arbitrary starting point in space (denoted by 0) in which 
the object, that is not under effect of any force, either appears to be at rest or in a state of uniform motion in a straight line. The position of the object is described with a position vector $(x, y, z)$ stretching from the starting point 0 to the object. This vector is a function of time, i.e. $(x(t), y(t), z(t))$. This vector and corresponding vectors of velocity and acceleration are decomposed into perpendicular directions of the coordinate system axes by projections onto relevant planes. Calculations describing various properties of movement are then completed separately for each plane defined by two of the axes.

We borrow this concept of decomposition and adapt it for our STC. The inertial frame of reference is replaced with the STC. We project each trajectory onto the $\mathrm{x}$-t and $\mathrm{y}$-t planes. These two projections are represented as two separate graphs that show how each object moves in one of the two geographic directions (figure 2). This approach is similar to the projection of time series data on to arbitrary planes developed within the GeoTime community [14]. These projected graphs can be used to visually investigate similarities of movement in each coordinate direction before applying the clustering techniques described below.

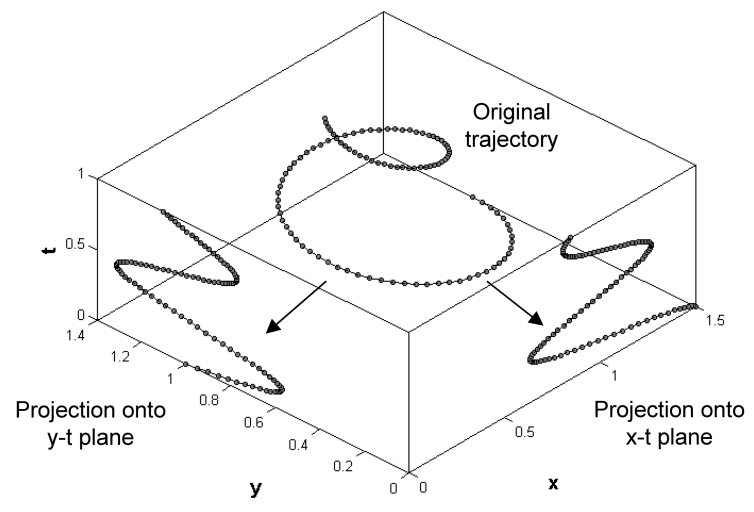

Fig. 2. Trajectories in a STC decomposed and projected on the $\mathrm{x}$-t and $\mathrm{y}$-t planes

\subsection{Trajectory Comparison}

To investigate similarity of trajectories we adopt a mathematical approach that looks at the shape of each projected curve. This can be done separately for each projection or by considering both projections simultaneously. Shape similarity is calculated based on the slope and rate of change of slope of a mathematical function in a graph. Similar approaches are used for time series [30], however there, only the slope of time series is utilised, while we also add the rate of change in slope for a more detailed approach. 
The first step in obtaining the required dataset is to invert the axes in each projection graph. This is done because the projected curves, as they are in a STC do not necessarily fulfill the criteria to form mathematical functions of a single variable, where at each value of the independent variable there should be at most one value of the dependent variable. Time cannot be given as a function of a single $\mathrm{x}$ or $\mathrm{y}$ coordinate as an object can visit the same location at different times. The inversion resolves this and is also logical from the conceptual sense of movement through time, since every object is always moving in a linear and unidirectional motion along this dimension. Similarity of two curves $x=f_{1}(t)$ and $x=f_{2}(t)$ in the t-x plane is then based on their slopes and rate of change in slope. Mathematically, the slope of a function $x=f(t)$ at a certain point $t$ is represented as the slope of the tangent to $f$ at that point, which is calculated as the first derivative at that point, i.e. slope $S=d f(t) / d t$. Since we derive over time, in physical terms this is equal to velocity of movement in $\mathrm{x}$ direction. Rate of change in slope is calculated as the second derivative of the function, i.e. change in slope $C=d f^{2}(t) / d t^{2}$. In physical terms, this is acceleration in $\mathrm{x}$ direction.

The shapes of two curves are more similar the more the slopes of the two curves (the derivatives) and the rate of change in slopes of the curves (the second derivatives) are similar to each other at each point $t$. Based on this principle, curves can be grouped into groups of similarly-shaped curves using the dataset of the first and second derivatives over time at all sampling times. Note, it is possible to compare either direction only ( $\mathrm{x}$ or $\mathrm{y}$ ) or both ( $\mathrm{x}$ and $\mathrm{y}$ ) in terms of first and second derivatives. For example, if the original trajectories are sampled from entities moving in a block-like city centre (e.g. Manhattan), then looking at each coordinate axis makes sense. If however objects move in a more unconstrained manner (e.g. hurricanes), then a joint dataset of derivatives in both $\mathrm{x}$ and $\mathrm{y}$ directions is a better option.

This principle does not consider the actual values on the curves (i.e. particular locations). The result is independent of the actual location of the moving objects. We are interested in studying movement behaviour, regardless of location. Furthermore, as the approach normalises time over each trajectory, it is not necessary for the trajectories being compared to be from the same period. The results of the procedure are therefore groups of trajectories that show similar behaviour, regardless of where and when the trajectories are positioned.

\subsection{Spectral Clustering}

Clustering, which involves identifying similarities between data points and using this as a basis to group them [31] is performed on the decomposed trajectories. Spectral Clustering [32], specifically the technique developed by Chen et al. [33] is used in the approach described here. Spectral Clustering has been effective for trajectory analysis [34], [35] however; the similarity measure was based on trajectory location, rather than movement behaviour, as in our case.

Spectral Clustering is a graph-based clustering technique. The graph is constructed by calculating a similarity matrix $S=s[i j]$ between all the data points 
in attribute space. We determine similarity using Euclidian distance between the first and second derivatives, as described in the previous section. An adjacency matrix of the similarity graph, $G=(V, E)$ is then produced, where $\mathrm{V}$ is the set of all data points, $x_{1} \ldots x_{n}$. Two vertices in the graph are connected only if the similarity between them $\left(s_{i j}\right)$, is larger than a given threshold value. With this approach, the complexity of original similarity matrix is reduced (making it sparse), as only significant similarities are considered. Clustering now involves partitioning the similarity graph into $k$ groups which can be determined through domain knowledge or analysis. Clusters are formed so that the edges between groups have a low similarity score while the edges within groups have a high similarity score. With Spectral Clustering, clusters are based on local similarity in the attribute space making this method faster. Additionally it can detect clusters that simple k-means would not recognise.

The groups resulting from the clustering process are then visualised. Firstly, the trajectories, which form each group, are plotted. The resulting graphs can explain the groupings and assist the analyst to apply expertise to classify entities based on the spatiotemporal movement patterns. The information is linked to the STC visualisation where individual clusters can be displayed for further visual analysis. The next section presents a case study which effectively applies these techniques to a real dataset.

\section{Case Study}

This section presents how the above approach is used to analyse pedestrian movement in the city of Delft, the Netherlands. Emphasis is placed on how the STC, summary plots, and associated analytics are used to infer knowledge and interpret the data. Data were collected over a 4 day period by distributing GPS receivers to pedestrians at car-park facilities in Delft. The receivers recorded the location of participants every 5 seconds. From the origin location, each participant made one journey on foot, represented by a single trajectory, from the origin, including stops, back to the origin which marks the end of a trip. Therefore, a trip can have multiple destinations and activities. The researchers who collected the data are interested in routes, destinations and sequences. This section demonstrates how temporal geovisualisation and clustering can answer these questions.

\subsection{Route Analysis}

A cleaned and validated set of trajectories representing the paths of 113 pedestrians was used to create the initial visualisation (figure 3) which highlights the spatiotemporal extent of the pedestrians. Each trip starts at ground level (time $0)$.

Through interaction, common spatial routes can be identified, for example, the majority of pedestrians travel east, while very few cross the barrier caused by railway tracks, west of the origin. Temporal analysis, achieved by examining 

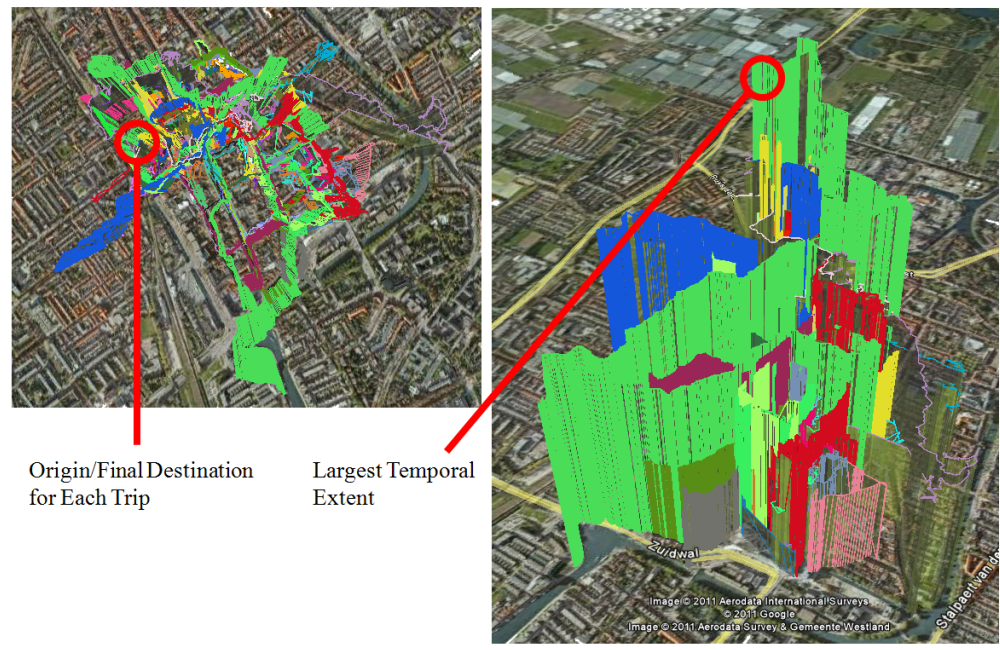

Fig. 3. All trajectories viewed from above and from the side in the STC showing the temporal and spatial elements of trajectories simultaneously.

the height of the trajectories on the time axis, shows that pedestrians generally travel in an anti-clockwise direction, visiting areas to the south of the origin first before regions, which are parallel with, or north of the origin. Domain knowledge indicates that pedestrians therefore visit chain stores before visiting boutiques.

The inclusion of temporal aspects of trajectories in the visualisation environment helps identify temporal co-existence which represents pedestrians who were travelling together. For example, trajectories which follow similar spatial and temporal routes are likely to be related. Examining figure 4, we can see two trajectories which initially follow this pattern. Midway through the trip, there is a split; one pedestrian continues walking while the other pedestrian stops at a single location. By examining the spatial content at the stop location, also shown in figure 4, it appears to be an office; a long stop here could indicate a meeting or an appointment. The ability to detect the spatial feature in this way demonstrates the power of the approach using Google Earth. Later, both trajectories exhibit a similar pattern again and return to the origin at a same time. Such behaviour seems to suggest that these pedestrians are travelling together and gives an indication of the social connections in the city.

Common locations where people stop provide evidence of salient areas [28]. Stopping locations are determined by the frequency of observations at the same location (based on a temporal threshold). Figure 5 shows all the stopping locations of pedestrians in this case study. Notable stopping locations are the two town squares where there are cafés and restaurants. The height of the rectangular prism indicates the duration of a stop which can distinguish between a stop at a restaurant and a visit to a shop perhaps. Multiple stopping locations on 


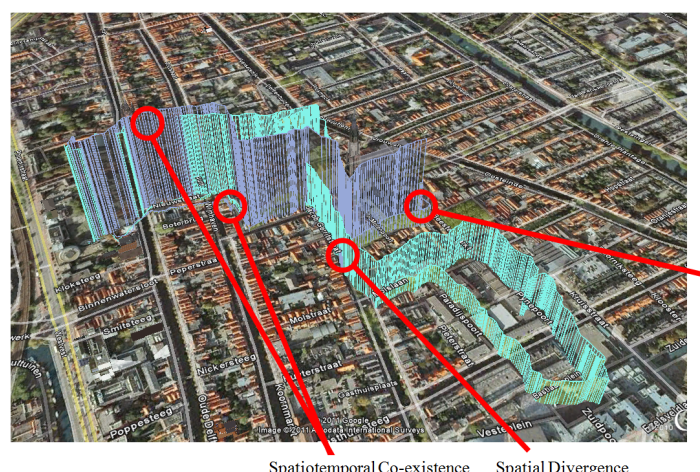

Spatiotemporal Co-existence Spatial Divergence

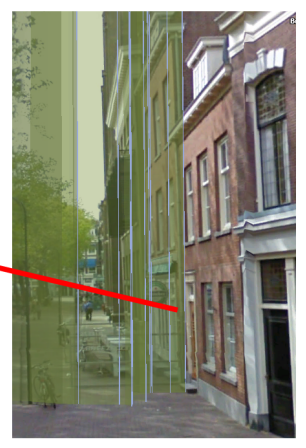

Location where one pedestrian stopped

Fig. 4. The STC showing two trajectories exhibiting spatiotemporal coexistence for a portion of their trips.

a particular route are significant. Figure 5 identifies the main shopping district based on the frequency and duration of stops which are indicative of people entering and leaving stores.

\subsection{Cluster Analysis}

Interactive graphs show the decomposed trajectories and are used to identify similarity among trajectories in each direction. As described in section 3.4, clustering is applied to the trajectories described in terms of speed and acceleration at each observation point, in each direction. Visual analysis suggested that 6 clusters produce descriptive results without overlap or gaps.

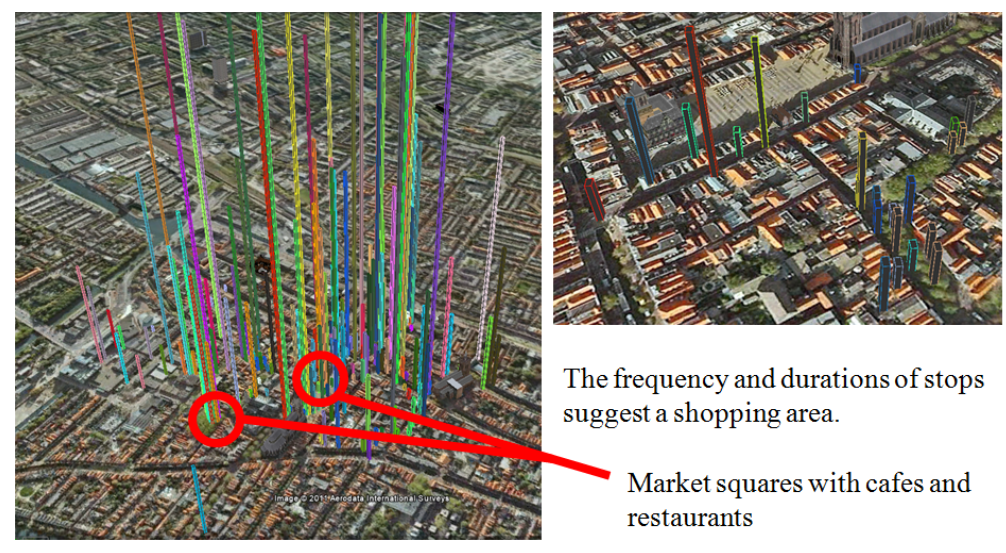

Fig. 5. The STC showing all identified stopping locations and the identification of a shopping area based on the duration and frequency of stops. 
Figures 6 - 11 show the graphs describing these clusters. Each graph has the same temporal extent to exemplify the differences between shorter and longer trips in each cluster. Time is represented on the $\mathrm{x}$-axis while the location is shown on the y-axis. Each cluster is represented by two graphs (latitude and longitude), however for the purpose of presentation; only one arbitrary graph for each cluster is shown here. Examining the graphs, it is clear to see how the temporal aspects of movement (duration, speed and acceleration) effect the clustering. The number of stops detected also plays an important role in classifying trajectories.

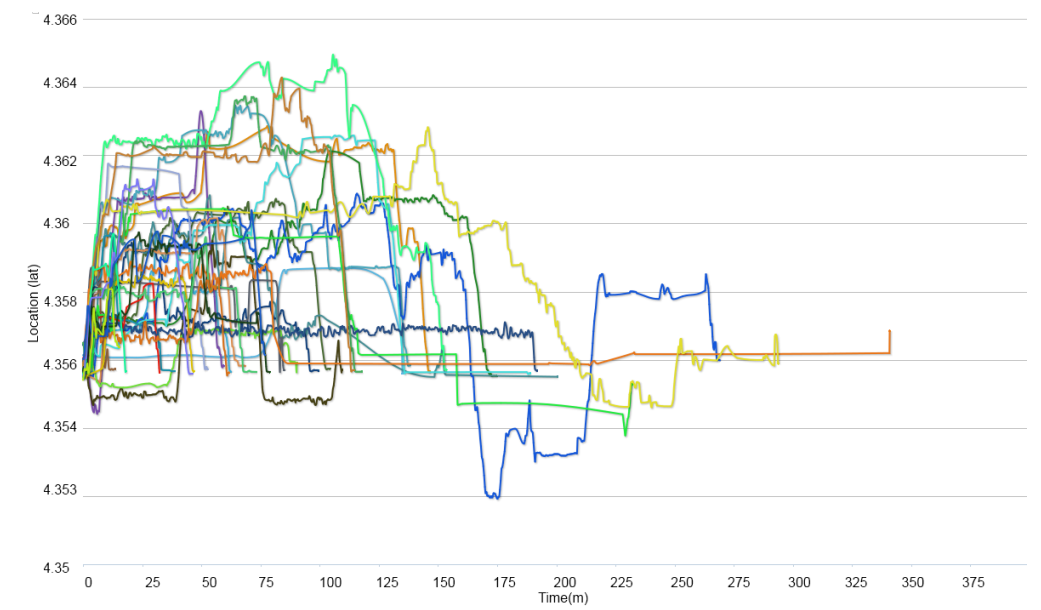

Fig. 6. Graph representing the trajectories in the longitude direction of cluster 1 . The cluster represents long trips with few stops.

When interpreting the graphs, the straight lines and high oscillating areas on a trajectory represent stop locations (oscillation occurs when a GPS receiver is stationary or the signal is lost when entering a building), while the smooth areas represent movement (figure 6). Using this knowledge, it is possible to describe the clusters in terms of movement patterns in Table 1. The trajectories, which form each cluster, can be visualised independently in the STC in order to gain further insight into pedestrian behaviour and determine if a spatial correlation also exists. For example, when the trajectories and stop locations for cluster 4, which contains a mix of medium and short length stops, is visualised using the STC, we can see pedestrians generally have their longest stop midway through their trip. While the trajectories within a cluster can be aggregated to find the most common trend or patterns, the geographic context is not considered during clustering and so visualising this aggregation in the STC is not necessarily beneficial. 


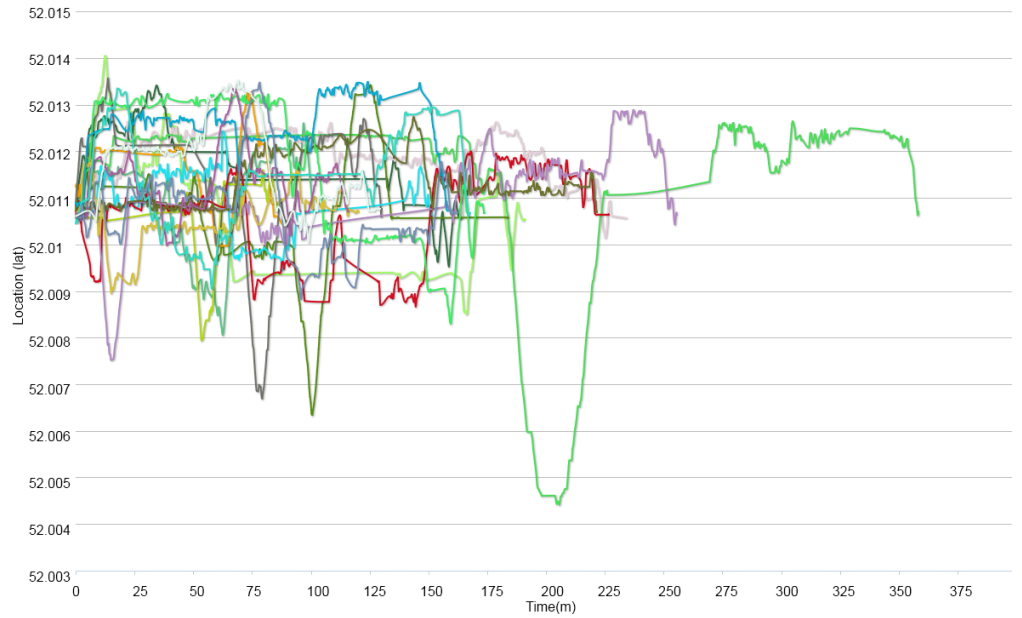

Fig. 7. Graph representing the trajectories in the latitude direction of cluster 2. The cluster represents long trips with many stops.

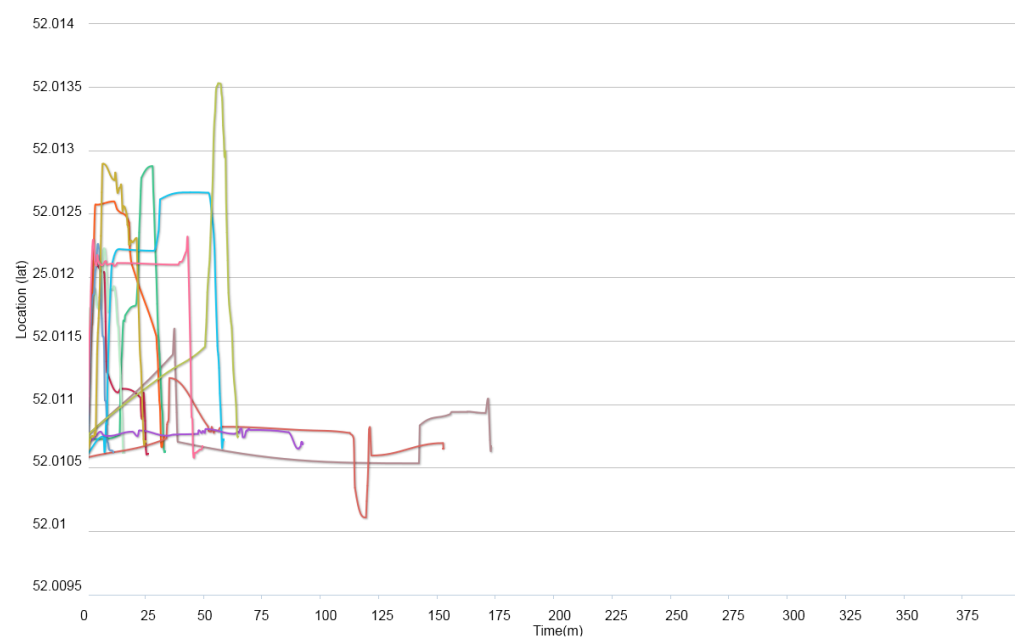

Fig. 8. Graph representing the trajectories in the latitude direction of cluster 3. The cluster represents short trips with no stops. 


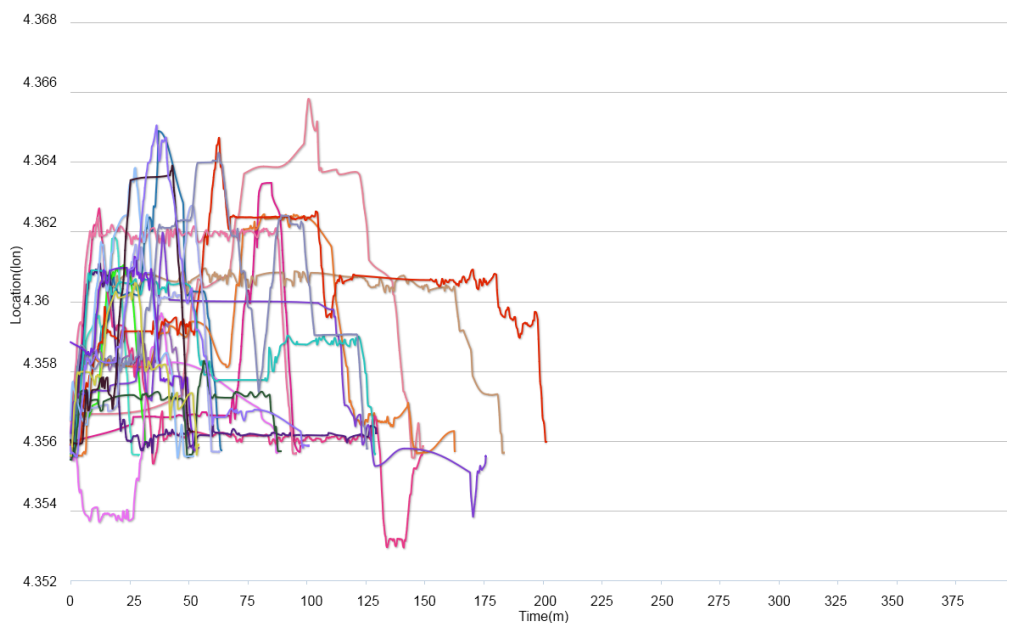

Fig. 9. Graph representing the trajectories in the longitude direction of cluster 4. The cluster represents medium length trips.

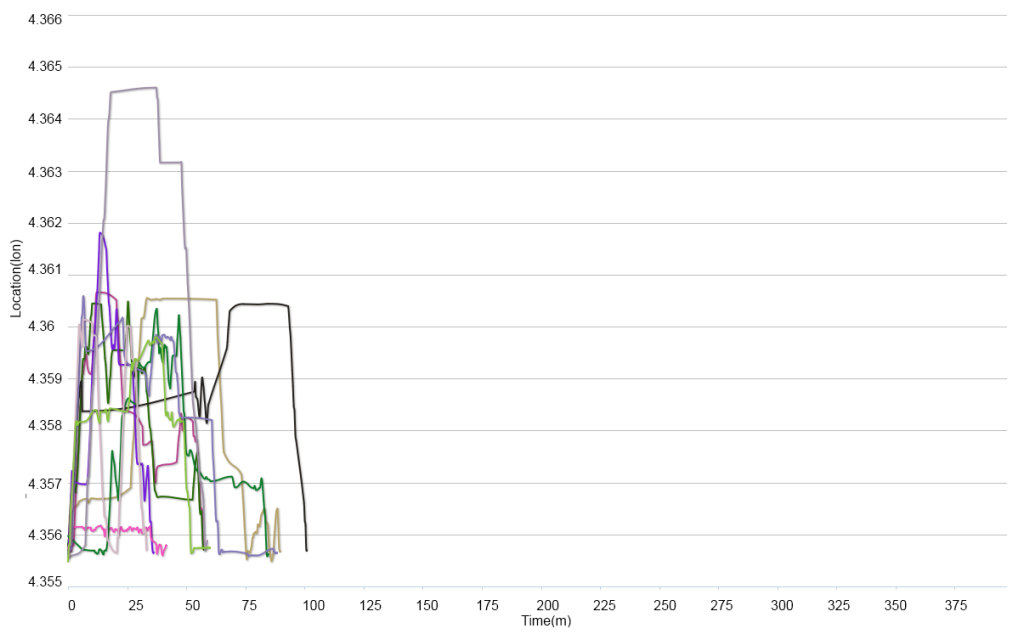

Fig. 10. Graph representing the trajectories in the longitude direction of cluster 5 . The cluster represents short trips with one stop. 


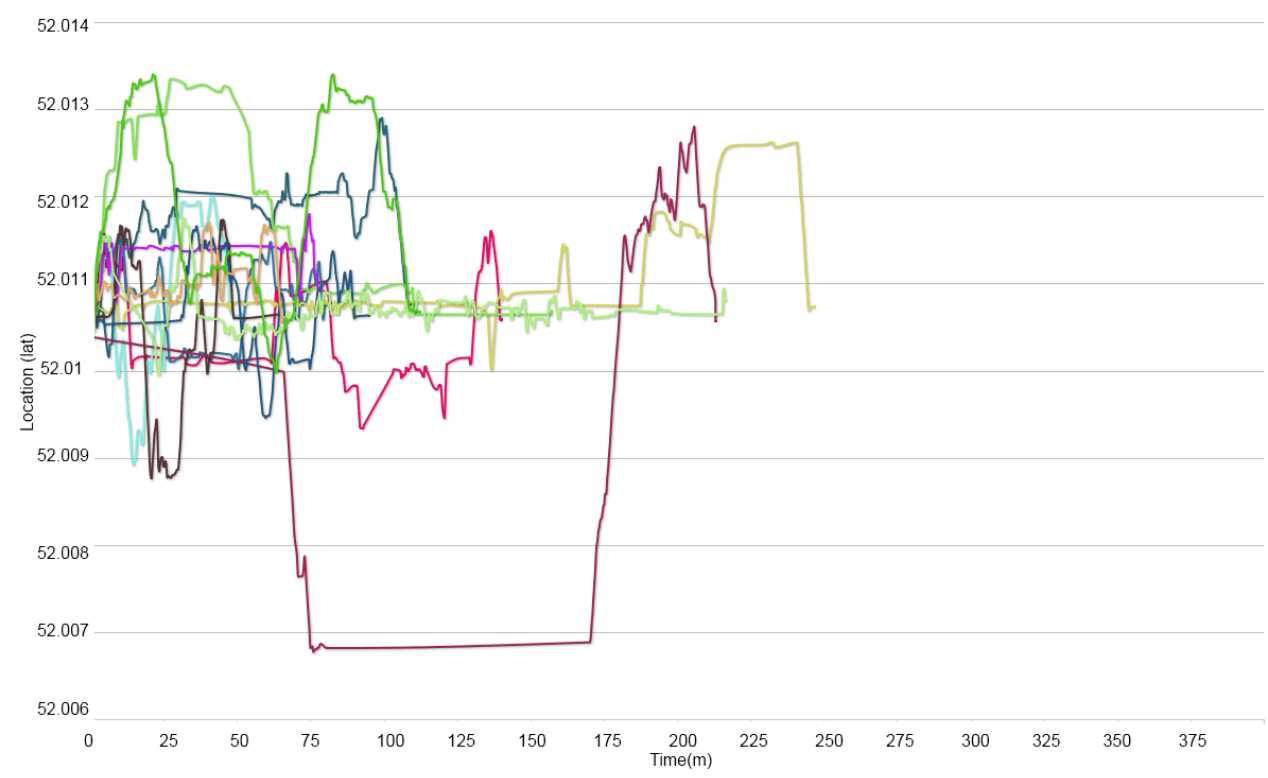

Fig. 11. Graph representing the trajectories in the latitude direction of cluster 6 . The cluster represents trips with one long stop.

\begin{tabular}{|c|c|c|c|c|}
\hline $\begin{array}{l}\text { Figure } \\
\text { No. }\end{array}$ & $\begin{array}{l}\text { Cluster } \\
\text { No. }\end{array}$ & Trip Duration & $\begin{array}{l}\text { Number of Stops/ } \\
\text { Stop Duration }\end{array}$ & Potential Explanation \\
\hline 6 & 1 & Long & Few/Long Stops & $\begin{array}{l}\text { Having lunch, a coffee or at- } \\
\text { tending a meeting }\end{array}$ \\
\hline 7 & 2 & Medium-Long & Many/Short Stops & Shopping \\
\hline 8 & 3 & Short & No Stops & $\begin{array}{l}\text { Wandering around the city } \\
\text { for brief window shopping }\end{array}$ \\
\hline 9 & 4 & Medium & $\begin{array}{l}\text { Many/Mix of short } \\
\text { and medium stops }\end{array}$ & $\begin{array}{l}\text { Shopping combined with } \\
\text { lunch/coffee }\end{array}$ \\
\hline 10 & 5 & Short & One/Short & $\begin{array}{l}\text { Specific purpose for trip - } \\
\text { shopping }\end{array}$ \\
\hline 11 & 6 & Medium & One/Long & $\begin{array}{l}\text { Specific purpose for trip - } \\
\text { meeting or lunch }\end{array}$ \\
\hline
\end{tabular}

Table 1. Summary of the clusters produced, along with a potential explanation of the behaviour detected 


\section{Discussion}

Although the approach is an effective visual analysis tool, there are several limitations which should be considered when interpreting the results. This section describes these limitations and indicates how future work can resolve many of them. One of the powers of Google Earth is the digital terrain model which shows the topography of the landscape. When analysing trajectories using the approach described above, analysts must be cognisant of the fact that the height of the trajectories is relative to the topography of the landscape. Analysts must examine the distance between the terrain (the Earth's surface) and the height of the trajectory at a given location to understand its temporal aspects. To aid with this, a shaded area between the Earth's surface and the trajectory has been included. The path of the trajectory may also be rendered along the Earth's contours to help interpret its relative height. In the case study described in this paper, we consider trajectories in the city of Delft in the Netherlands which is a flat geographical region and so this issue is not apparent.

The clustering approach described here projects each trajectory into its latitude and longitude components to visually identify similarity in movement patterns. While this may be considered an arbitrary choice, it is based on the fact that this is how movement is generally recorded and described. While trajectories can be described by many different attributes, in this study, we are interested in the behaviour of walking pedestrians who are not necessarily in the same spatial area. Velocity and acceleration are therefore good proxies for this form of behaviour as stops and moves can be used to gauge similarity in spatially disjoint regions.

The clustering technique produced six clusters. As is the case with most clustering techniques applied to real-world data, assessing the validity of the cluster assignments is not possible without some ground truth. While the pedestrians in the case study were asked if they were going shopping or visiting tourist locations, this information did not translate to the richer data revealed in the clusters. In future studies, questions can be tailored to match cluster outputs.

\section{Conclusion}

Movement is a fundamental aspect of the physical world. Studying movement data and recognising patterns can be utilised in many domains to predict future movement, assess how spaces are used and identify unusual behaviour. This article introduces a powerful geovisual environment, for exploring movement data. The concept of a classic STC is extended and used in conjunction with Google Earth so that the temporal and spatial aspects of trajectories can be analysed simultaneously. The approach adheres to the classic visual information seeking mantra by providing an 'Overview first', followed by 'Zoom and filter', and 'Details-on-demand' paradigm [36].

Similarity among trajectories is determined by describing them in terms of the speed and acceleration at each observation point. Euclidean distance in the 
attribute space of first and second derivatives then determines similarity before Spectral Clustering is applied to produce groups. The techniques are particularly valuable for detecting trajectories which have a similar duration and contain a similar number of stops but are not necessarily in the same geographic location.

The approach was tested by exploring pedestrian trajectories in a city. While pedestrian movement and city life have been investigated before, existing approaches are generally based on counting and local observations without insight into complete activity patterns [37]. Knowing more about how people use the city, not only as individuals in a specific situation, but also translated to general situations based on the clusters of pedestrians produced, interventions to improve the city can be proposed, for example by identifying barriers that cause an imbalance between frequently visited and neglected places.

In the case study, the clustering of pedestrian trajectories results in 6 groups of pedestrians. While the descriptions of the clusters are indicative of activities undertaken by pedestrians in a city centre, the ground truth regarding the actual activities and intentions of those pedestrians would be beneficial to validate the clusters. This will be carried out in a future study and the results will be used to enhance the clustering technique.

\section{Acknowledgements: Removed for Review}

\section{References}

1. S. Dodge, R. Weibel, and E. Forootan. Revealing the physics of movement: Comparing the similarity of movement characteristics of different types of moving objects. Computers, Environment and Urban Systems, 33(6):419-434, 2009.

2. S. Orlando, R. Orsini, A. Raffaetà, A. Roncato, and C. Silvestri. Trajectory data warehouses: design and implementation issues. Journal of Computing Science and Engineering, 1(2):240-261, 2007.

3. A. Millonig and G. Gartner. Identifying motion and interest patterns of shoppers for developing personalised wayfinding tools. Journal of Location Based Services, $5(1): 3-21,2011$.

4. X. Zheng, T. Zhong, and M. Liu. Modeling crowd evacuation of a building based on seven methodological approaches. Building and Environment, 44(3):437-445, 2009.

5. S.P. Hoogendoorn and P.H.L. Bovy. Pedestrian travel behavior modeling. Networks and Spatial Economics, 5(2):193-216, 2005.

6. S. Spek. Tracking tourists in historic city centres. Information and Communication Technologies in Tourism 2010, pages 185-196, 2010.

7. J. Van Schaick. Timespace matters-exploring the gap between knowing about activity patterns of people and knowing how to design and plan urban areas and regions. 2011.

8. P. Laube, S. Imfeld, and R. Weibel. Discovering relative motion patterns in groups of moving point objects. International Journal of Geographical Information Science, 19(6):639-668, 2005.

9. C. Wilson. Activity patterns in space and time: calculating representative hagerstrand trajectories. Transportation, 35(4):485-499, 2008. 
10. S. Dodge, R. Weibel, and A.K. Lautenschütz. Towards a taxonomy of movement patterns. Information visualization, 7(3-4):240-252, 2008.

11. G. Andrienko and N. Andrienko. Spatio-temporal aggregation for visual analysis of movements. In Visual Analytics Science and Technology, 2008. VAST'08. IEEE Symposium on, pages 51-58. IEEE, 2008.

12. T. Hägerstrand. What about people in regional science? Papers in Regional Science, 24(1):6-21, 1970.

13. M.J. Kraak. The space-time cube revisited from a geovisualization perspective. In Proc. 21st International Cartographic Conference, pages 1988-1996, 2003.

14. T. Kapler and W. Wright. Geotime information visualization. Information Visualization, 4(2):136-146, 2005.

15. M.J. Kraak. Geovisualization and time-new opportunities for the space-time cube. Geographic visualization: concepts, tools and applications, pages 293-306, 2008.

16. G. Andrienko, N. Andrienko, and S. Wrobel. Visual analytics tools for analysis of movement data. ACM SIGKDD Explorations Newsletter, 9(2):38-46, 2007.

17. U. Demšar and K. Virrantaus. Space-time density of trajectories: exploring spatiotemporal patterns in movement data. International Journal of Geographical Information Science, 24(10):1527-1542, 2010.

18. G. Andrienko and N. Andrienko. Poster: Dynamic time transformation for interpreting clusters of trajectories with space-time cube. In Proceedings of the IEEE Symposium on Visual Analytics Science and Technology (VAST) 2010, pages 213$214,2010$.

19. J.S. Horne, E.O. Garton, S.M. Krone, and J.S. Lewis. Analyzing animal movements using brownian bridges. Ecology, 88(9):2354-2363, 2007.

20. A. Slingsby, J. Strachan, P.L. Vidale, J. Dykes, and J. Wood. Discovery exhibition: Making hurricane track data accessible. Discovery Exhibition Entry, 2010.

21. E. Frentzos, K. Gratsias, N. Pelekis, and Y. Theodoridis. Algorithms for nearest neighbor search on moving object trajectories. Geoinformatica, 11(2):159-193, 2007.

22. Z. Zhang, K. Huang, and T. Tan. Comparison of similarity measures for trajectory clustering in outdoor surveillance scenes. In Pattern Recognition, 2006. ICPR 2006. 18th International Conference on, volume 3, pages 1135-1138. IEEE, 2006.

23. K. Buchin, M. Buchin, and C. Wenk. Computing the fréchet distance between simple polygons in polynomial time. In Proceedings of the twenty-second annual symposium on Computational geometry, pages 80-87. ACM, 2006.

24. Y. Sakurai, M. Yoshikawa, and C. Faloutsos. Ftw: fast similarity search under the time warping distance. In Proceedings of the twenty-fourth ACM SIGMODSIGACT-SIGART symposium on Principles of database systems, pages 326-337. ACM, 2005.

25. M. Vlachos, G. Kollios, and D. Gunopulos. Discovering similar multidimensional trajectories. In Data Engineering, 2002. Proceedings. 18th International Conference on, pages 673-684. IEEE, 2002.

26. L. Chen, M.T. Özsu, and V. Oria. Robust and fast similarity search for moving object trajectories. In Proceedings of the 2005 ACM SIGMOD international conference on Management of data, pages 491-502. ACM, 2005.

27. D. Ashbrook and T. Starner. Using gps to learn significant locations and predict movement across multiple users. Personal and Ubiquitous Computing, 7(5):275286, 2003.

28. A.T. Palma, V. Bogorny, B. Kuijpers, and L.O. Alvares. A clustering-based approach for discovering interesting places in trajectories. In Proceedings of the 2008 ACM symposium on Applied computing, pages 863-868. ACM, 2008. 
29. D. Halliday, R. Resnick, and J. Walker. Fundamentals of Physics. Wiley, 1997.

30. F. Altiparmak, H. Ferhatosmanoglu, S. Erdal, and D.C. Trost. Information mining over heterogeneous and high-dimensional time-series data in clinical trials databases. Information Technology in Biomedicine, IEEE Transactions on, 10(2):254-263, 2006.

31. A.K. Jain, M.N. Murty, and P.J. Flynn. Data clustering: a review. ACM computing surveys (CSUR), 31(3):264-323, 1999.

32. Y. Song, W.Y. Chen, H. Bai, C.J. Lin, and E. Chang. Parallel spectral clustering. Machine Learning and Knowledge Discovery in Databases, pages 374-389, 2008.

33. W.Y. Chen, Y. Song, H. Bai, C.J. Lin, and E.Y. Chang. Parallel spectral clustering in distributed systems. Pattern Analysis and Machine Intelligence, IEEE Transactions on, 33(3):568-586, 2011.

34. S. Atev, G. Miller, and N.P. Papanikolopoulos. Clustering of vehicle trajectories. Intelligent Transportation Systems, IEEE Transactions on, 11(3):647-657, 2010.

35. B. Morris and M. Trivedi. Learning trajectory patterns by clustering: Experimental studies and comparative evaluation. In Computer Vision and Pattern Recognition, 2009. CVPR 2009. IEEE Conference on, pages 312-319. IEEE, 2009.

36. B. Shneiderman. The eyes have it: A task by data type taxonomy for information visualizations. In Visual Languages, 1996. Proceedings., IEEE Symposium on, pages 336-343. IEEE, 1996.

37. J. Van Schaick and S.C. Van Der Spek. Urbanism on track: Application of tracking technologies in urbanism, volume 1. Ios PressInc, 2008. 